\title{
Heart Rate Variability Analysis and The Influence of Exercise Intensity Over Time in Young-healthy Women
}

\author{
Aisha Widi Rahayu ${ }^{1}$, Izza Alifa Hassya ${ }^{1}$, Eki Dipo Laksono ${ }^{1}$, and Alvin Sahroni ${ }^{*}$, \\ ${ }^{I}$ Electrical Engineering Department, Faculty of Industrial Technology, Universitas Islam Indonesia, Yogyakarta, Indonesia \\ *Corresponding Author: alvinsahroni@uii.ac.id
}

\begin{abstract}
Our heart is a vital organ that pumps blood and through the vessels of the circulatory system. In medical applications, we can observe the heart rate using Electrocardiograph (ECG). Currently, people tend to have high working activity without a proper exercise intensity. This study was conducted to observe the heart rate variability (HRV) on the healthy young woman who was not doing any exercise. We evaluated the HRV characteristics while exercising with a regular period and different intensity (light to hard) and how the difference before and after of evaluation period. Seven young-healthy women (19 - 21 years old) women were observed during three observation stages: pre-exercise, main exercise-period, and post-exercise for 2 months. We analyzed MeanRR, SDRR, CVRR, rMSSD, VLF, LF, HF, and the Poincaré plot parameters (SD1 and SD2) as the HRV properties. We found that SDRR was decreased from the first week $(0.08 \mathrm{~s})$ to the last week of the evaluation period $(0.03 \mathrm{~s})$ followed by the HF component $(0.15-0.2 \mathrm{~Hz})$. The Poincaré plot properties also reduced from the first week to the last week of the exercise period $(0.07 \mathrm{~s}$ to $0.03 \mathrm{~s})$. We indicated the characteristics of a woman's HRV during regular exercise periods with different intensity have made the heart more effective in pumping blood. We concluded that the heart condition would be improved during regular exercise with the increment of intensity even in a short of a period. Finally, the heart rate performance may be decreased during absent from regular exercise for a month.
\end{abstract}

Keywords: Heart Rate Variability (HRV), exercise intensity, young-healthy women, ECG

Article History: Received 1 July 2020; Accepted 20 March 2021; Available online: 29 March 2021

DOI: $10.14710 /$ jbiomes.2021.v1i1.1-6

\section{INTRODUCTION}

As we already know that the heart is a special pump that contracts for the delivery of blood throughout the body [1]. The pumping action is based on the electricity flows through the heart and repeats itself in a cycle that we know as heart rate or heart pulse [2]. Heart rate can be interpreted as the speed of the heartbeat measured by the number of contractions per unit of time, and it can be determined by computing the heart rate variability (HRV). Heartbeat is defined as the work of the heart muscle that occurs between contraction (systole) and relaxation (diastole). The frequency of the heartbeat shows how fast the heart pumps blood through the circulatory system. Along with the heart rate, measurements of arterial pressure can indicate the health of a person measured as an initial reference for a doctor to determine the health condition of a patient [3].

A heart attack is one of the deadliest causes of the disease, which also called a silent killer. Regular exercise can help to maintain the human heart health and manage the cardiovascular system more efficient in pumping blood and delivering oxygen to the muscles. Therefore, the mechanism of the release of adrenaline and lactic acid to the blood will increase heart rate [3]. Currently, we already know that the HRV was able to represent the autonomic nervous function and improving the diagnose of medical application. HRV can be measured by recording heart data from an Electrocardiograph (ECG) device. Using an ECG, we not only record the heart rate signal but we also able to observe how much variation in heart rate can be known. This variation of heart rate is a reference in diagnosing human health conditions [4].

The utilization of HRV and how it can be used to assess the cardiovascular autonomic parameters is still ongoing and suggested as an important study. It also used as an essential method for assessing cardiovascular autonomic parameters that represent the control regulation of the autonomic nervous system (sympathetic and parasympathetic modulation) $[5,6]$. By evaluating the HRV through the ANS regulation, it is possible to evaluate the function of the cardiovascular system to recognize healthy and diseased states due to the vagal-mediated HRV indices were inversely associated with several risk factors (diabetes, glucose, intolerance, insulin resistance, central obesity, dyslipidemia, and hypertension) [7]. While HRV is largely applied to diagnose cardiac diseases, recent studies demonstrated the application of HRV in exercise training [8 - 11]. Their findings supported the use of HRV as a marker to represent the ANS modulation, and it is suggested that monitoring indices of HRV during several time courses of training or adaptation/maladaptation could improve exercise performance $[7,11,12]$.

In the previous studies, they found that the effect of exercises was able to increase the blood pressure caused by routine physical training on heart performance [8]. Nonetheless, a study by Etzel found the interests of the differences of exercise between men and women, $40 \%$ of women not doing any exercise or physical activities. In addition, only $30 \%$ of men not doing any exercises [9]. Regarding this issue, the idea arises to analyze the effect of exercise intensity on heart rate variability in young-healthy 
women who not regularly doing any exercises. Specifically, we would like to demonstrate how the routine with incremental intensity could affect the cardiac properties and how the difference between pre- and post-exercise regarding the HRV parameters. Our hypothesis is the HRV properties changed during time, and our study could contribute to expanding the knowledge of sports physiology related to an exercise program to improve a healthy cardiovascular system.

\section{MATERIALS AND METHODS}

\subsection{Research Ethics}

This research has obtained informed consent from all subjects involved. This research has been attempted to follow the Declaration of Helsinki research that regulates the rules when research relates to human subjects.

\subsection{Subjects}

Seven subjects participated in this study. The subjects' age was between 18-22 years. All subjects should have a normal BMI scale, same height, not smoke or vape, and not routinely exercise of any type. Subjects should refrain from consuming drugs, food, or drinks that can trigger the cardiovascular system, and no heart disease history. A Healthy criterion means that the subject in a normal condition according to its function. There are no diseases disorder and can run daily activities normally. We obtained that information by conducting a health condition questionnaire or history of the disease given to the subject before the study was conducted. The subject's normal BMI has criteria sourced from the Indonesian Ministry of Health with the criteria of women; skinny: $<17 \mathrm{~kg} / \mathrm{m} 2$, normal: $17-$ $23 \mathrm{~kg} / \mathrm{m} 2$, obesity: $23-27 \mathrm{~kg} / \mathrm{m} 2$, obesity:> $27 \mathrm{~kg} / \mathrm{m} 2$ [4]. Subject BMI calculations were assisted using the BMI calculator application. The subject was also given informed consent before the study was conducted. Subjects who are not willing to fill out the Informed Consent will not continue to be the subject of this research.

\subsection{Experimental Design}

The experiment conducted during the standing position. We were using ATTYS, a bio-signal acquisition device, and the sampling frequency was set to $250 \mathrm{~Hz}$. This proposed study was using the ECG limb leads II placement system. Subjects were standing during data recording, and they were not allowed to talk or move their body since the beginning of the experiment. The length of the ECG data retrieval was set to twenty minutes.

The type of applied exercise is the jump rope. Recorded data were separated into three parts/sessions. The first part was a week before the implementation of the regular exercise (Pre-Exercise). The second part was the day after the physical activity or exercise periods (conducted three times a week for two months). During the physical activity's routine, the jumping intensities were increased 25 jumps every week until the end of physical activity periods with
200 jumps in total. The third part of the experiment was the post-exercise period that taken a month after the exercise periods.

\subsection{Signal Processing and Features Extraction}

We established ECG peaks detection algorithm to extract the features. We detect and extracted the R-R peaks that came from the heartbeat to beat interval for 20 minutes recording. Then we calculated the time and frequency domain parameters. Also, we include the Poincaré Plot analysis to represent the non-linear analysis method.

The time-domain analysis consists of the mean of R-R intervals duration (MeanRR), the standard deviation of the beats intervals (SDRR), the ratio between SDRR and MeanRR (CVRR), and the root means square of standard deviation (rMSSD). The frequency-domain analysis is observing the component frequency by using Fourier transform frequency decomposition of the R-R time series that divided into Very Low Frequency/VLF (0.0033 - 0.04 $\mathrm{Hz})$, Low Frequency/LF (0.04 - $0.15 \mathrm{~Hz})$, and High Frequency/HF $(0.15-0.4 \mathrm{~Hz})$.

The Poincaré Plot analysis observes the R-R interval that plotted against the previous R-R intervals. The center point of the plot represents the average R-R interval length of the plot. The interval lengths are longitudinal and transverse lines. The length of the transverse lines is defined as the SD1. The SD2 is perpendicular to the transverse line that we called the longitudinal line. The Poincare index SD1 and SD2 were computed.

\subsection{Data Analysis}

We employed the non-parametric method to analyze the data and observed the difference between exercise periods. The data were presented using mean and standard deviation (SD). We also examine the correlation between subjects' data to find the relationship of physiological parameters changes with the exercise periods. The probability value was set to 0.05 , which means that if the p-value less than 0.05 , the comparative analysis considered different significantly.

\section{RESUlts}

Seven participants were joined this study and their cardiac properties were observe over 2 months. This study demonstrated the HRV parameters were able to represent cardiac modulation changes over time courses on a younghealthy woman who not able to exercise regularly. The time and frequency domain, and non-linear methods consistently show the trend changes from the beginning of the experiment until post-exercise.

Fig. 1 shows an example of HRV indexed on a subject that there were changes that occurred every week with the increment of jumps. It was indicated by the variations of R$\mathrm{R}$ intervals tend to decrease from the $1^{\text {st }}$ week to the $8^{\text {th }}$ week, respectively. The interesting finding that the HF components were decreased, especially the frequency around $0.15-0.2$ $\mathrm{Hz}$. However, the pre-post conditions show no significant difference both in time and frequency domain. 

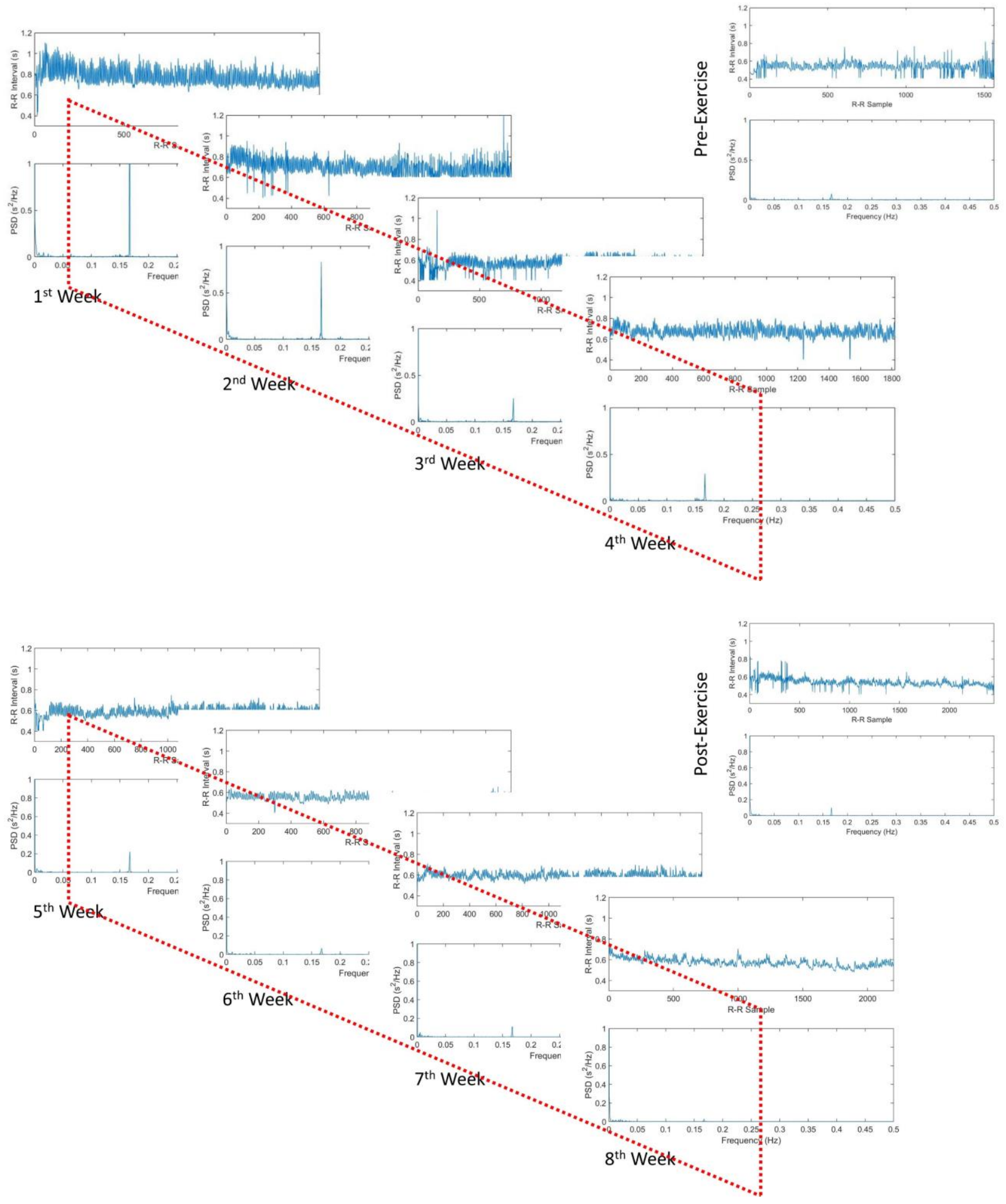

Fig. 1 The R-R intervals time series and the frequency components tend to decrease over time

According to Poincaré Plot analysis that represents a non-linear method to reflect the cardiac properties, it is depicted in Fig. 2 that HRV properties changes occurred in each week observation as same as the time and frequency domain. The results were indicated by the elliptical pattern of the Poincaré plot (SD1 \& SD2). In the Poincaré plot, the $1^{\text {st }}$ week parameters show the deviation from the Poincare $\mathrm{R}-$ $\mathrm{R}$ interval is around $0.7 \mathrm{~s}-0.86 \mathrm{~s}$. The $2^{\text {nd }}, 3^{\text {rd }}, 4^{\text {th }}, 5^{\text {th }}, 6^{\text {th }}$ , and the $8^{\text {th }}$ week are around $0.65 \mathrm{~s}-0.75 \mathrm{~s}, 0.55 \mathrm{~s}-0.63 \mathrm{~s}$, $0.63 \mathrm{~s}-0.70 \mathrm{~s}, 0.55 \mathrm{~s}-0.63 \mathrm{~s}, 0.50 \mathrm{~s}-0.56 \mathrm{~s}, 0.58 \mathrm{~s}-0.60 \mathrm{~s}$, and $0.55 \mathrm{~s}-0.60 \mathrm{~s}$ respectively. Pre-exercise is in the range of $0.50 \mathrm{~s}-0.58 \mathrm{~s}$, and the Post-exercise was in the range of 


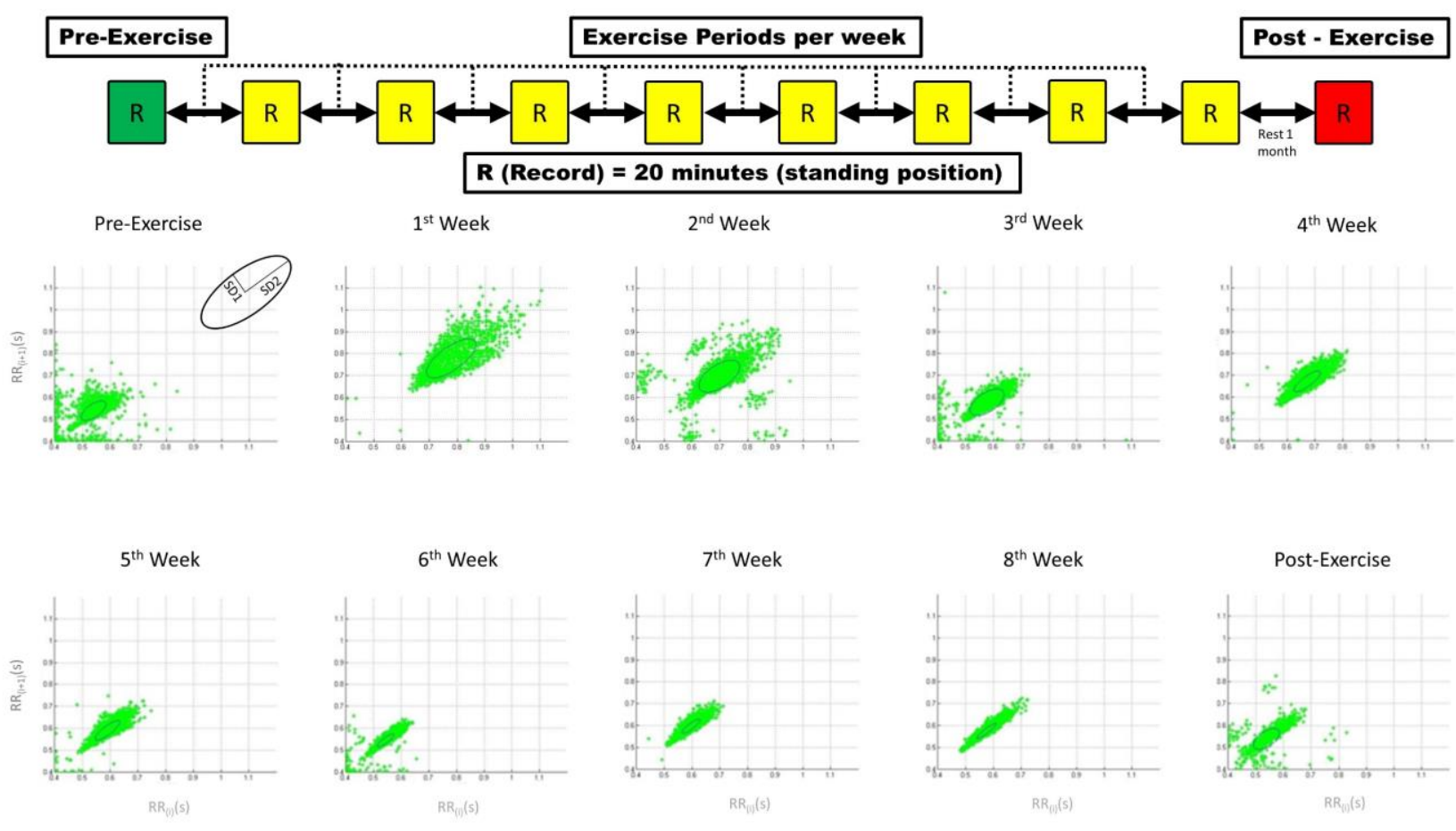

Fig. 2 The Poincaré Plot analysis in different weeks of exercise

$0.50 \mathrm{~s}-0.60 \mathrm{~s}$. The elliptic size that shows the longitudinal and transverse length also shows a reduced tendency along different weeks. As same as the time and frequency domain, the tendency shows the same properties that reflect the cardiac parameters are changed over 2 months exercise routine with incremental intensity every week.

According to Fig. 1 and 2, we could identify that the variation of R-R intervals plays a big-role to differentiate the cardiac modulation changes during 2 months of exercise routine with different intensities each week. As already mentioned in the previous section that the variation or deviation of $\mathrm{R}-\mathrm{R}$ time intervals could reflect the cardiac properties and explain how the cardiac modulation as well. To strengthen these findings, we established the SDRR index evaluation over seven young-healthy women during pre-post exercise and during the eight weeks experiment.

Based on Fig. 3(a)., it shows us that there were consistent patterns that occurred in all subjects. The variation of R-R intervals or the SDRR index tends to decrease, and these alterations are identical to the participants from the first week to the last week of exercise periods. Also, there is a high deviation of R-R interval in the first week compared with other weeks when the exercise began to be applied to all subjects.

The chart in Fig. 3(b) shows the pre-post subjects' SDRR parameter to reflect cardiac changes. It is depicted that the pre- and post-condition are decreased during exercise. Preexercise SDRR is higher than post-exercise, but it does not occur in subject 7 . However, the statistics show that the results indicated the data were not showing any difference at the beginning of the exercise compared to post-exercise.
Based on the time-frequency domain, and geometrical analysis from Poincaré plot characteristics, we found that the SDRR index is the most sensitive parameter that reflects the cardiac changes compared to other parameters. According to Fig. 3(c), there are significant differences shown at $1^{\text {st }}$ week to $2^{\text {nd }}, 3^{\text {rd }}, 4^{\text {th }}$, and $5^{\text {th }}$ week. There also significant differences between $2^{\text {nd }}$ week with the $3^{\text {rd }}, 4^{\text {th }}$, and $5^{\text {th }}$ week, followed by the $3^{\text {rd }}$ week with $4^{\text {th }}, 5^{\text {th }}, 6^{\text {th }}$ week. In addition, the significant difference also found between $5^{\text {th }}$ week to the $6^{\text {th }}$ week. However, there is no significant differences in $7^{\text {th }}$ and $8^{\text {th }}$ week.

To reinforce the SDRR as an important parameter in this study, we examine the consistency of SDRR parameter on every subject by correlating the parameter with different time courses. All data on SDRR have a high correlation value ( $p$-value $<0.05$ ) except for the comparison between subject 5 and subject 2 , and between subject 5 and subject 4 . This parameter has a high correlation test, seen only two data

Table 1. The correlation between subjects regarding the SDRR parameter in different exercise time courses

\begin{tabular}{|c|c|c|c|c|c|c|c|}
\hline & S1 & S2 & S3 & S4 & S5 & S6 & S7 \\
\hline S1 & 1 & & & & & & \\
\hline S2 & 0.826 & 1 & & & & & \\
\hline S3 & 0.915 & 0.810 & 1 & & & & \\
\hline S4 & 0.873 & 0.980 & 0.844 & 1 & & & \\
\hline S5 & 0.794 & 0.640 & 0.707 & 0.684 & 1 & & \\
\hline S6 & 0.877 & 0.749 & 0.838 & 0.836 & 0.706 & 1 & \\
\hline S7 & 0.942 & 0.888 & 0.850 & 0.949 & 0.804 & 0.884 & 1 \\
\hline
\end{tabular}




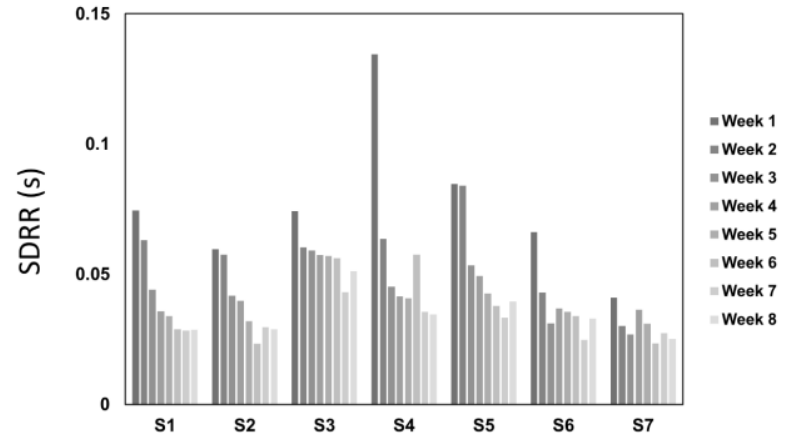

(a)

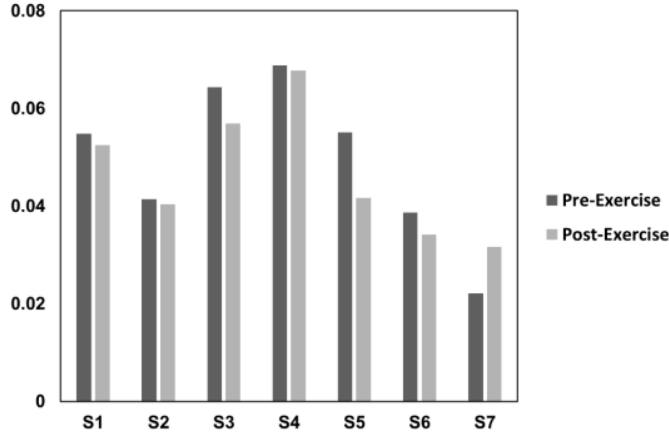

(b)

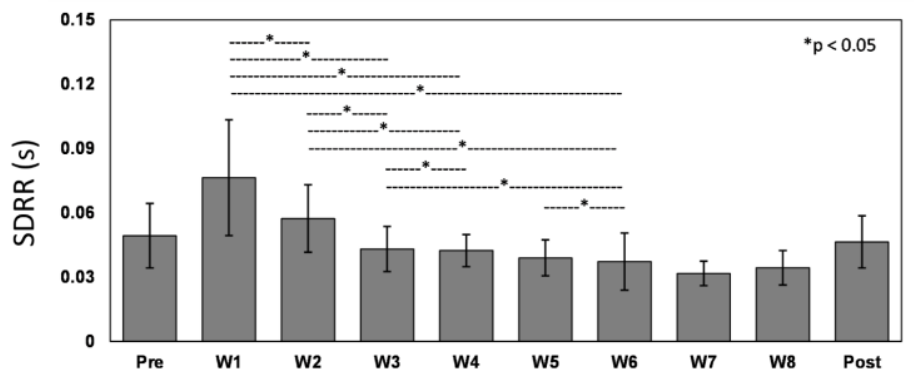

(c)

Fig. 3 SDRR parameter as the most informative cardiac properties to represent physiological changes during exercise

that do not correlate. It can be concluded that the SDRR parameter is a feature that able to show the correlation of HRV changes from the first week to the last week in each subject.

\section{Discussions}

\subsection{HRV Exercise Periods and Pre-Post Exercise}

The R-R interval's data already showed in Fig. 1 and 2 demonstrated the changes that occurred during the exercise periods with incremental intensity each week. Fig. 1 depicted that there is a pattern or trend that decrease every week during the period of regular exercise. Not only the time domain properties but also the frequency domain in the specific frequency range. The changes also can be seen in Fig. 2, which the size of the ellipse of Poincaré Plot analysis (SD1 \& SD2) tends to shrink, both the longitudinal and transverse lines every week as well. This phenomenon showed that cardiac properties are changed when exercise is routinely carried out as a training effect [13]. Besides, there is a high $\mathrm{R}-\mathrm{R}$ intervals value in the first week when the exercise began to be applied. That is because there is a process of bodily adaptation, especially in the part of the heart, as the cardiac system that makes adjustments that initially never did exercise at the beginning of the study. After doing some routine exercise with different intensity, the characteristics of the heart also change with time [14,15].

Even though Fig. 3(a) and 3(c) shows that the heart rate characteristics reduced over time, the tendency seems to approach the initial cardiac conditions before the experiment begins. During a month's absence of exercise routine, we can see that the variability of cardiac changes during pre- and post-exercise are not much different. Regarding those results, we found that each week's transition strongly related to the improvement of the cardiovascular profile. The previous study reported that both aerobic and anaerobic moderates physical training, and it can improve the cardiovascular characteristic due to the decrement of heart rate properties in a specific population [14].

To ascertain changes in heart conditions, hypothesis testing, and correlation tests on HRV data were obtained. Based on Fig. 3, the SDRR parameter provides the highest difference compared to other cardiac indexes. These findings can be seen from the number of exercise weeks that have pvalues of the statistical test less than 0.05. Also, the correlation test performed in Table 1 convinces with mostly have a high correlation and the overall results that we already obtained reflect the physiological changes as the effect of the training, and most informed by SDRR parameter.

\subsection{Physiological Changes}

SDRR parameter is the most informative parameter that show the physiological changes in the heart that occurred during this study. The decrease in the trend that occurs from the first week to the last week shows that physiological changes in the heart when exercise is routinely carried out. Besides, there are high R-R intervals values in the first week when the exercise began to be applied. This surge in SDRR can be caused by the physiological adaptation response of a person's body during routine exercise, and endurance training will cause many changes to the cardiovascular system. The heart rate changes on the SDRR parameter that have decreased indicates that the heart's performance in pumping blood is getting more maximal. This is because the 
excessive activity of the muscles during exercise will stimulate other body systems. This excessive muscle contraction requires the metabolic system to provide more energy for the continuation of muscle contraction. This also stimulates the cardiovascular system to provide oxygen needs while distributing the residue of metabolism. In order to complete the needs of energy, metabolism in the muscles will increase. This increase in metabolism requires a source of energy and oxygen; at the same time, it will also be followed by the removal of metabolic waste in the muscles.

Increased demand and residue of metabolism require the cardiovascular system to increase blood flow to the muscles. The decrease in SDRR every week shows that the heart rate has decreased or has slowed when routine exercise is applied. Decreasing or slowing heart rate is caused by the enlargement of the systolic ventricular volume and the contents of the heart stroke, which has an impact on the increased capacity or ability of the heart to drain blood. In a medical journal said that regular physical exercise for long periods would lead to increased efficiency of cardiovascular function. Reduction in pulse frequency, physiological heart enlargement, and decreased peripheral resistance are forms of this efficiency [3]. Besides, results were obtained (Fig. 2), and show an increase in the trend that occurred at $8^{\text {th }}$ week on subject 1 , subject 2, subject 3, and subject 4. Most likely, an increase in trend occurred due to the maximum ability of body adaptation in exercise.

\section{Conclusion}

The HRV characteristics of the young-healthy woman during regular exercise for two months with incremental jumps intensities have made the heart more effective in pumping blood. According to the SDDR index, it shows that the SDRR value tends to decrease in different weeks. The SDRR parameters are decreased from the highest value during the first week of exercise until the last week of the experiment $(0.08 \mathrm{~s}$ to $0.03 \mathrm{~s})$, and it is also similar from the frequency domain in the HF component $(0.15-0.2 \mathrm{~Hz})$, and the Poincaré plot as well. The correlation test also shows that inter-subject analysis mostly shows a strong relationship between the cardiac indexes and the changes in the exercise time courses. Finally, the cardiac properties may be decreased when a person absent from exercise as long as needed.

\section{ACKNOWLEDGMENTS}

This work is supported by the Electrical Engineering Department, Faculty on Industrial Technology, Universitas Islam Indonesia annual research funding for final project student research.

\section{REFERENCES}

[1] Boudoulas, Konstantinos Dean, Ioannis A. Paraskevaidis, Harisios Boudoulas, and Filippos K. Triposkiadis. "The left atrium: from the research laboratory to the clinic." Cardiology vol. 129, no. 1, pp. 1-17, 2014.

[2] Acharya, U. Rajendra, K. Paul Joseph, Natarajan Kannathal, Choo Min Lim, and Jasjit S. Suri. "Heart rate variability: a review." Medical and biological engineering and computing, vol. 44, no. 12, pp. 1031-1051, 2006.

[3] Canadian Society for Exercise Physiology, "Canada's Physical Activity Guide to Healthy Active Living," Public Heal. Agency Canada, 1998.

[4] R. Almeida, S. Gouveia, A. P. Rocha, E. Pueyo, J. P. Martínez, and P. Laguna, "QT variability and HRV interactions in ECG: Quantification and reliability," IEEE Trans. Biomed. Eng., vol. 53, no. 7, pp. 13171329, 2006.

[5] Khandoker, Ahsan H., Herbert F. Jelinek, and Marimuthu Palaniswami. "Identifying diabetic patients with cardiac autonomic neuropathy by heart rate complexity analysis." Biomedical engineering online, vol. 8, no. 1 , pp. 3, 2009

[6] Tereshchenko, Larisa G., Iwona Cygankiewicz, Scott McNitt, Rafael Vazquez, Antoni Bayes-Genis, Lichy Han, Sanjoli Sur et al. "Predictive value of beat-to-beat QT variability index across the continuum of left ventricular dysfunction: competing risks of noncardiac or cardiovascular death and sudden or nonsudden cardiac death." Circulation: AR-Rhythmia and Electrophysiology, vol. 5, no. 4, pp. 719-727, 2012.

[7] Hemingway, HaR-Ry, Martin Shipley, Eric Brunner, Annie Britton, Marek Malik, and Michael Marmot. "Does autonomic function link social position to coronary risk? The Whitehall II study." Circulation, vol. 111 , no. 23 , pp. 3071-3077, 2005 .

[8] Syatria, Arsdiani." The Effects of Programmed Sport Exercise on Blood Pressure of Students of Diponegoro University Faculty of Medicine who Joined The Basketball Team (Pengaruh olahraga terprogram terhadap tekanan darah pada mahasiswa Fakultas Kedokteran Universitas Diponegoro yang mengikuti ekstrakurikuler basket)." PhD diss., Faculty of Medicine, Universitas Diponegoro, 2006.

[9] R. A. Etzel, "Women Physicians Among Authors of Perspective-Type Articles Published in High-Impact Pediatric Journals," JAMA Netw. Open, vol. 1, no. 3, p. e180899, 2018.

[10]Zimatore, G., M. C. Gallotta, L. Innocenti, V. Bonavolontà, G. Ciasca, M. De Spirito, L. Guidetti, and C. Baldari. "Recurrence quantification analysis of heart rate variability during continuous incremental exercise test in obese subjects." Chaos: An Interdisciplinary Journal of Nonlinear Science, vol. 30, no. 3, pp. 033135, 2020.

[11]Urzeală, Constanta, Aura Bota, Sorin Serbanoiu, Mariana Mezei, Frederic Dutheil, and Daniel Courteix. "Heart rate variability as a possible predictor of sport performance in junior rhythmic gymnastics." Isokinetics and Exercise Science Preprint, pp. 1-9, 2020.

[12] Pham, Quoc Cuong, Tran Duc Minh Nguyen, Cao Dang Le, Quoc Khai, and Quang Linh Huynh Le. "Exercise Physiology: Improving Stationary Bike Training Performance Using Heart Rate Variability." Kalpa Publications in Engineering, vol. 3, pp. 100-108, 2020).

[13] Hottenrott, Kuno, Olaf Hoos, and Hans Dieter Esperer. "Heart rate variability and physical exercise. Current status." Herz, vol. 31, no. 6, pp. 544-552, 2006.

[14]Eula, E., F. Abate Daga, C. Fulcheri, S. Di Monaco, M. Pappaccogli, E. Perlo, E. Fanelli et al. "Cardiovascular Changes Due To Physical Activity In Obese And/Or Hypertensive Children: A Prospective Cohort Study." Journal of Hypertension, vol. 37, pp. e179, 2019.

[15] Routledge, F.S., T.S. Campbell, J.A. McFetridge-Durdle, and S.L. Bacon. "Improvements in heart rate variability with exercise therapy." Canadian Journal of Cardiology, vol. 26, no. 6, p.303-312, 2010. 